\title{
Disharmony between wake- and respiration-promoting activities: effects of modafinil on ventilatory control in rodents
}

Jiro Terada ${ }^{1,2^{*}}$, Isato Fukushi ${ }^{2,3}$, Kotaro Takeda $^{2,4}$, Yohei Hasebe ${ }^{2,5}$, Mieczyslaw Pokorski ${ }^{2,6}$, Koichiro Tatsumi $^{1}$ and Yasumasa Okada²

\begin{abstract}
Background: Modafinil is a wake-promoting drug and has been widely used for daytime sleepiness in patients with narcolepsy and other sleep disorders. A recent case series reported that daily oral modafinil alleviated hypercapnic respiratory failure in patients with COPD. However, the precise action of modafinil on respiration such as hypercapnic and/or hypoxic ventilatory responses remains unclear. The aim of this study is to clarify the effect of modafinil on the ventilatory control.

Methods: We investigated the hypothesis that modafinil enhances resting ventilation as well as the stimulatory ventilatory responses to hypercapnia and hypoxia. We addressed the issue by examining minute ventilation, respiratory rate and volume components using plethysmography, combined with a concurrent EEG monitoring of the level of wakefulness before and after administration of modafinil in two doses of $100 \mathrm{mg} / \mathrm{kg}$ and $200 \mathrm{mg} / \mathrm{kg}$ in unanesthetized mice. In addition, we monitored the effect of the lower dose of modafinil on mice locomotor activity in a freely moving condition by video-recording.
\end{abstract}

Results: Wakefulness, locomotor activity and variability of the breathing pattern in tidal volume were promoted by both doses of modafinil. Neither dose of modafinil increased the absolute values of resting ventilation or promoted the ventilatory responses to hypercapnia and hypoxia. Rather, higher dose of modafinil slightly suppressed respiratory rate in room air condition.

Conclusions: Modafinil is conducive to the state of wakefulness but does not augment resting ventilation or the hyperventilatory responses to chemical stimuli in unanesthetized rodents.

Keywords: Breathing, Electroencephalogram, Hypercapnic ventilatory response, Hypoxic ventilatory response, Narcolepsy, Respiratory failure, Vigilance, Wake-promoting drug

\section{Background}

Modafinil is a widely-used wake-promoting drug for treating somnolence in narcolepsy and residual sleepiness persisting in obstructive sleep apnea despite continuous positive airway pressure treatment [1-3]. The drug activates brain areas involved in the control of arousal state through dopaminergic, orexinergic $[4,5]$, and histaminergic [6] neurotransmitter pathways, and the sympathetic

\footnotetext{
* Correspondence: jirotera@chiba-u.jp

'Department of Respirology, Graduate School of Medicine, Chiba University,

1-8-1 Inohana, Chuo-ku, Chiba city, Chiba 260-8670, Japan

${ }^{2}$ Clinical Research Center, Murayama Medical Center, 2-37-1 Gakuen,

Musashimurayama, Tokyo 208-0011, Japan

Full list of author information is available at the end of the article
}

nervous system. Recent studies on modafinil, in addition to a reduction in daytime sleepiness, have also demonstrated a spate of neurological effects such as cognitive enhancement and antidepressant effects $[7,8]$. These multifarious actions of modafinil led us to hypothesize that it could affect ventilation [3].

The process of setting the ventilatory drive originates in the brain stem and is entwined with the central neural pathways above outlined, although this is an area of limited understanding. Notably, however, ventilation and its stimulatory responses to hypercapnia and hypoxia assume a depressive vein in sleep; the condition which modafinil is expected to counteract. It was thus a 
reasonable assumption that modafinil would enhance ventilation and its responses. The assumption was strengthened by a recent report demonstrating that modafinil, given orally on a daily basis, alleviated hypercapnic respiratory failure in COPD patients (i.e., improving oxygenation, and lowering the arterial carbon dioxide level) without adverse effects [9]. This off-label use of modafinil opens a new door to regulate hypercapnic respiratory failure in noncompliance patients with non-invasive ventilation [10]. However, the precise action of modafinil on the respiratory control system has not been fully investigated.

Additionally, traditional wake-promoting agents such as methylxanthines [11], methylphenidate [12], and amphetamine [13] have been known to cause some positive influence on respiratory control. Among those, methylxanthines have been clinically used, and their mechanisms of action have been well analyzed. For example, caffeine is frequently used for the treatment of apnea in premature infants, and also enhances the ventilatory response to hypoxia [14]. Theophylline and aminophylline prevent hypoxic ventilatory depression via inhibition of adenosine $[15,16]$. Methylphenidate is known to induce emergence from general anesthesia by increasing arousal and respiratory drive [12].

In this study we seek to determine the effects of modafinil on resting ventilation in room air and on the stimulatory hypercapnic/hypoxic ventilatory responses in unanesthetized spontaneously breathing mice. We monitored the arousing central effects of modafinil by recording electroencephalogram (EEG) and by video-recording locomotor activity. In addition, the effect of modafinil on blood gas content was checked in unanesthetized spontaneously breathing rats. In contrast to our presumption, the investigation failed to demonstrate any appreciable effect of modafinil on ventilatory regulation.

\section{Methods \\ Animals}

Nine conscious, spontaneously breathing, male C57BL/6 mice (age $16.5 \pm 0.6$ weeks, weight $26.9 \pm 0.6 \mathrm{~g}$ ) were used in the study of ventilatory measurement. Further, to analyze arterial blood gas, six conscious, spontaneously breathing, male Wistar rats (age $25.0 \pm 0.1$ weeks, weight $284 \pm 7 \mathrm{~g}$ ) were used. The mice and rats were housed in separate cages at $23-24{ }^{\circ} \mathrm{C}, 50-60 \%$ relative humidity, and $12 / 12 \mathrm{~h}$ light/dark cycle, and were fed with commercial chow and water ad libitum. All experiments were conducted in sleep-time for rodents $(9 \mathrm{am}-5 \mathrm{pm})$.

\section{Experimental preparation Recording of EEG}

To monitor the functional status of the forebrain in mice, EEG was recorded. Surgical procedures were described in our previous report [17]. Briefly, EEG electrodes were implanted on the skull under isoflurane anesthesia, followed by intraperitoneally injected pentobarbital. The skull was exposed and three miniature screws were inserted; two were over the frontal lobes $2.5 \mathrm{~mm}$ posterior to the bregma as recording electrodes, and one was along the midline $4.5 \mathrm{~mm}$ anterior to the bregma as a ground electrode. The mice were allowed to recover from surgery for at least 1 week until EEG recordings began. EEG signals were amplified (JB-101 J and AB-651 J, Nihon Kohden, Tokyo, Japan) and bandpass filtered in $0.08-100 \mathrm{~Hz}$ frequency range.

\section{Recording of ventilation}

Resting ventilation, and the hypoxic and hypercapnic ventilatory responses were measured in a whole body rodent plethysmograph (PLY 310, EMMS, Bordon, UK) consisting of the recording (volume $530 \mathrm{~mL}$ ) and reference chambers, as previously described [17-20]. Briefly, the chambers were placed inside a transparent acrylic box (size $20 \times 20 \times$ $20 \mathrm{~cm}$ ). Each mouse was placed in the pre-calibrated recording chamber. Chamber temperature was maintained constant at $25{ }^{\circ} \mathrm{C}$ throughout the experiment. The air in the recording chamber was suctioned with a constant flow generator. To calculate the respiratory flow, the pressure difference between the recording and reference chambers was measured with a differential pressure transducer (TPF100, EMMS), connected to an amplifier (AIU060, Information \& Display Systems, Bordon, UK), and was bandpass filtered at $0.1-20 \mathrm{~Hz}$. The signal was integrated to obtain tidal volume $\left(\mathrm{V}_{\mathrm{T}}[\mu \mathrm{L} /\right.$ weight (gram)]) for each respiratory cycle, which was then averaged throughout the period of interest. Respiratory rate (RR [breaths/min]) was counted. Minute ventilation $\left(\dot{\mathrm{V}}_{\mathrm{E}}[\mathrm{mL} / \mathrm{g} / \mathrm{min}]\right)$ was calculated as $V_{T} \times R R$. To evaluate the effect of modafinil on variability of the breathing pattern, the coefficients of variation of $\mathrm{V}_{\mathrm{T}}$ and total respiratory cycle (a reciprocal of RR) were calculated as described in a previous study [21]. The $\mathrm{O}_{2}$ concentration in the chamber was continuously monitored with an $\mathrm{O}_{2}$ analyzer incorporating a polarographic sensor (Respina IH 26, San-ei, Tokyo, Japan), and was adjusted by controlling the mixing of nitrogen gas and air flows blown into the acrylic box. The pressure and EEG signals, together with $\mathrm{O}_{2}$ concentration data were simultaneously digitalized at $400 \mathrm{~Hz}$ sampling with an A/D converter (PowerLab4/26, ADInstruments, Colorado Springs, $\mathrm{CO}$ ) and stored in a PC with LabChart7 software (ADInstruments).

\section{Experimental protocols for whole body plethysmography}

Ventilation and its responses to sequential inspired gas changes before and after administration of a wakepromoting agent, modafinil were recorded in the tethering condition with EEG. The sequence of experimental 
steps is shown in Fig. 1. The hypercapnic $\left(5 \% \mathrm{CO}_{2}\right)$ ventilatory response was analyzed in a hyperoxic condition to eliminate the hypoxic influence. The hypoxic ventilatory response was analyzed by loading $10 \% \mathrm{O}_{2}$. Each gas mixture was maintained in the chamber for $5 \mathrm{~min}$. Our experimental protocol of gas exchange (e.g., the pattern and length of gas exposures) was based on a previous report, which showed "time domain of ventilatory response" [22], and our previous studies, which adopted a protocol similar to the present study $[17,19,20]$. Because a concern was raised that resting ventilation or the hypoxic ventilatory response may be affected when hypoxic exposure was repeated. However, in our pilot study ( $n=5$, male C57BL/6 mice, age 15-17 weeks) repeating similar hypoxic exposure (see Fig. 1 ) with vehicle injection three times (DMSO trial 1-3) showed that ventilation was not confounded by the repeated hypoxic exposure, i.e., hypoxic ventilatory depression did not appear (see Additional file 1). Thus, the prior gas exposures should not have affected the ventilatory responses to the succeeding gas exposures in the present study. Modafinil (Sigma-Aldrich, St. Louis, MO) was dissolved with dimethyl sulfoxide (DMSO), and injected intraperitoneally in a volume of $0.5 \mathrm{ml} / \mathrm{kg}$. After injection, 50 min was allowed for acclimation to a plethysmograph chamber and the following $10 \mathrm{~min}$ were taken as the baseline level of ventilation before the introduction of gas changes. Each exposure trial started in $60 \mathrm{~min}$ after injection of modafinil since previous research indicated peak response of modafinil in rodents especially for wakefulness is in 60-180 min after the injection $[6,23,24]$. This protocol was repeated, with intervals at least $10 \mathrm{~min}$, in the following sequence:

(1)DMSO alone $(0.5 \mu \mathrm{L} / \mathrm{g})$

(2) modafinil (lower dose, $100 \mathrm{mg} / \mathrm{kg}$ ) in DMSO $(0.5 \mu \mathrm{L} / \mathrm{g})$

(3) modafinil (higher dose, $100 \mathrm{mg} / \mathrm{kg}$, cumulative dose $200 \mathrm{mg} / \mathrm{kg})$ in DMSO $(0.5 \mu \mathrm{L} / \mathrm{g})$.

The total dose of DMSO was $1.7 \mathrm{mg} / \mathrm{kg}$, which does not affect respiratory function [20]. Although the doses of modafinil were certainly different from that used in humans, yet we used these two doses. This was because $50-300 \mathrm{mg} / \mathrm{kg}$ of modafinil adequately affects wakefulness and other physiological activities in rodents $[4,24,25]$.

\section{Blood gas analysis}

Under anesthesia with isoflurane followed by pentobarbital injection $(40 \sim 50 \mathrm{mg} / \mathrm{kg}$, i.p.), a polyethylene catheter (Tombo No.9003 PFA, inner diameter $0.5 \mathrm{~mm}$, outer diameter $1.0 \mathrm{~mm}$, Nichias, Tokyo, Japan) was inserted into a unilateral femoral artery in rats to sample arterial blood. The catheter was filled with saline containing heparin, tunneled subcutaneously, and exteriorized at the dorsal midline between the bilateral scapulae. The exteriorized catheter tip was covered with a jacket to protect from biting by the rats. After catheterization, the rats were allowed to recover for at least $48 \mathrm{~h}$. Then, DMSO and modafinil was injected in the following sequences same as the mice protocol: (1) DMSO alone, (2) modafinil (lower dose, $100 \mathrm{mg} / \mathrm{kg}$ ) in DMSO, (3) modafinil (higher dose, $200 \mathrm{mg} / \mathrm{kg}$ ) in DMSO. In blood gas analysis experiments, modafinil purchased from Alfresa Pharma (Osaka, Japan) was used. Sixty minutes after each injection, arterial blood $(0.2 \mathrm{~mL})$ was sampled, and arterial blood gas was analyzed (Blood Gas Analyzer ABL77, Radiometer, Copenhagen, Denmark). After each sampling of arterial blood, saline was intraarterially injected to maintain circulating blood volume constant.

\section{Video monitoring}

To monitor the effect of modafinil on mice locomotor activity in a freely moving condition, behavior of mice in an ordinary cage was video-recorded before and after intraperitoneal administration of modafinil $(100 \mathrm{mg} / \mathrm{kg})$.

\section{Data analyses}

In the measurement of ventilation, periods during which the mouse moved (e.g., sniffing, grooming, and licking) were excluded, but these periods were counted as locomotor activity (\% period) in mice tethered with the EEG device. Data were given as mean \pm standard error. The mean values of $V_{T}, R R, \dot{V}_{E}$, the coefficient of variations $\left(\mathrm{V}_{\mathrm{T}}\right.$ and total respiratory cycle) and locomotor activity

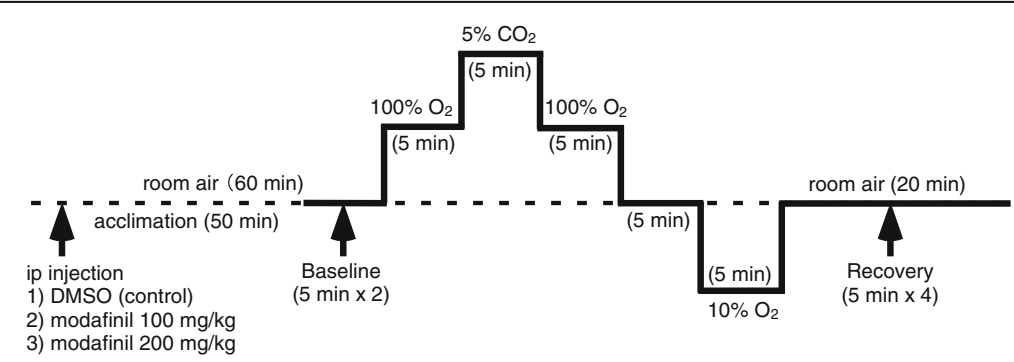

Fig. 1 Scheme of the experimental protocol. See Methods for details of inspired gas mixture changes 
were respectively submitted to a two factor withinsubject analysis of variance (ANOVA), with three pharmacological conditions; vehicle and two doses of modafinil, and four air conditions; baseline room air, hypercapnia $\left(5 \% \mathrm{CO}_{2}\right.$ in $\left.\mathrm{O}_{2}\right)$, hypoxia $\left(10 \% \mathrm{O}_{2}\right.$ in $\left.\mathrm{N}_{2}\right)$, and recovery room air. Whenever necessary, a GreenhouseGeisser adjustment was used to correct for violations of sphericity. We applied Bonferroni correction for the multiple comparisons in post-hoc test. Statistical significance was set at $P<0.05$. The signal processing was performed using MATLAB 2015a (MathWorks, Natick, MA) and all statistical analyses were performed using SPSS 22.0 (IBM, NY).

\section{Results}

Unceasing restlessness was observed in eight out of the nine mice approximately $30 \mathrm{~min}$ after administration of modafinil $(100 \mathrm{mg} / \mathrm{kg})$. One mouse, injected with the higher dose modafinil $(200 \mathrm{mg} / \mathrm{kg})$, died after the experimental protocol of hypoxic challenge was completed.

\section{Wakefulness and locomotor activity}

A change in EEG signal to low amplitude and relatively high frequency was noted after injection of modafinil in the mice (Fig. 2). Locomotor activity was clearly promoted after injection of both low and high doses of modafinil (Fig. 3). The increase in locomotion was about the same for both doses of modafinil; the higher dose did not potentiate the effect. Nor were there any major changes in locomotion during consecutive gas mixtures swaps in the chamber, with a tendency for locomotion stimulation during hypoxia after modafinil. Video monitoring for the mice without EEG attachment in an ordinary cage showed that the movement of mouse injected with modafinil (right cage) was continuously activated compared with the control mouse (left cage) (see Additional file 2).

\section{Respiration}

The respiratory flow signal at rest changed to more irregular pattern along with promoted wakefulness after both doses injection of modafinil in the mice (see Fig. 2). Coefficient of variation of $\mathrm{V}_{\mathrm{T}}$ (but not total respiratory cycle) was significantly enhanced in mice with modafinil, especially with higher dose of modafinil (Fig. 4). However, any absolute level of ventilation (i.e., either $V_{T}, R R$, or $\dot{V}_{E}$ ) was not promoted after modafinil injection, compared with the control injection of DMSO (Fig. 4). In contrast, baseline $V_{T}$, baseline and recovery $R R$ in room air decreased after administration of higher doses of modafinil; recovery $\dot{V}_{\mathrm{E}}$ decreased with the higher dose of modafinil with a similar tendency of baseline $\dot{\mathrm{V}}_{\mathrm{E}}(P=0.051)$. Modafinil, in either dose, failed to appreciably affect ventilation during exposure to hypercapnia or hypoxia. The only exception was a slight but significant suppressive effect of modafinil on RR during the hypercapnic response observed at the lower dose of modafinil; with a similar tendency at the higher dose (Fig. 4). Note that tidal volume as shown in Fig. 4 was calculated as cumulative sum of respiratory flow in whole body plethysmography, but not solely determined by the magnitude of peak flow as shown in Fig. 2.

Arterial blood gas values $\left(\mathrm{Pa}_{\mathrm{O} 2}, \mathrm{~Pa}_{\mathrm{CO} 2}\right.$ and $\left.\mathrm{pH}\right)$ in six rats did not change after injection of modafinil, compared with control (Fig. 5).

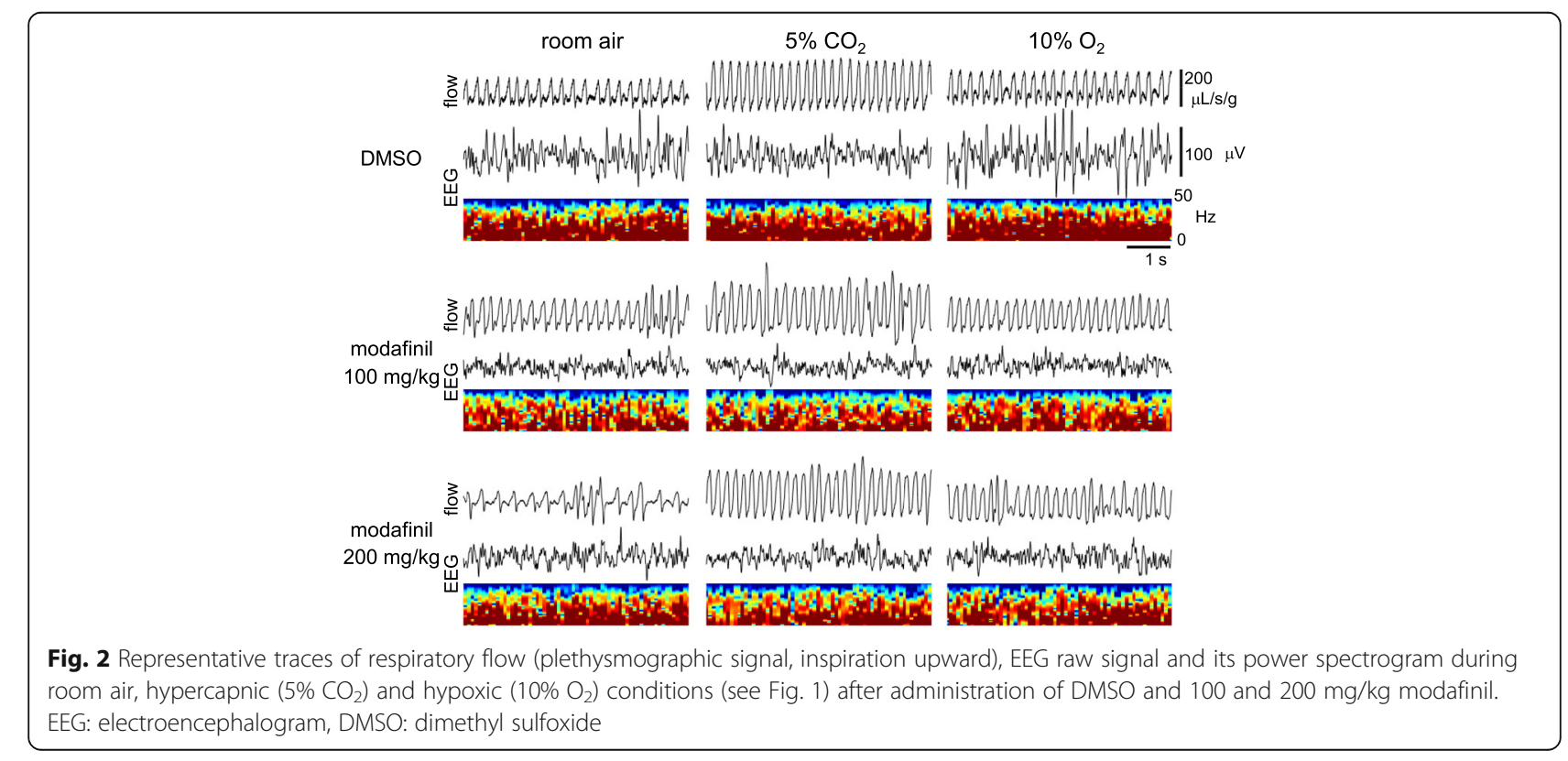




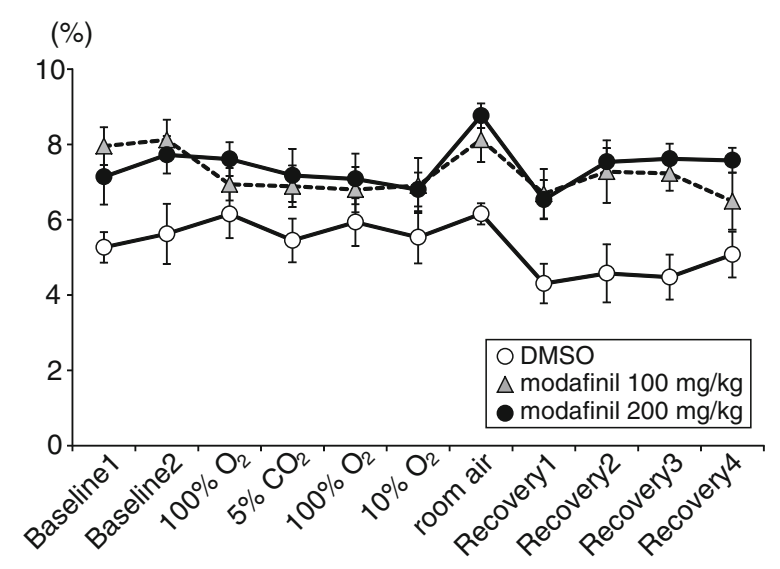

Fig. 3 Locomotor activity during experimental protocols (see Fig. 1) in three pharmacological conditions: DMSO and 100 and $200 \mathrm{mg} / \mathrm{kg}$ modafinil. Five minutes were allocated in each gas change protocol (e.g., Baseline1, Baseline2, 10\% $\mathrm{O}_{2}$ ). Significant differences among the three pharmacological conditions $(F(2,16)=9.53, P<0.01)$ and the four air conditions $\left(F(3,24)=7.50, \varepsilon_{G G}=0.57, P<0.01\right)$ were revealed by the two-way repeated measures ANOVA. However, interaction was not significant $(F(6,48)=1.20, P>0.05)$. DMSO: dimethyl sulfoxide. Baseline: room air condition, Recovery: room air condition

\section{Discussion}

The major finding of this study is that modafinil, a wakepromoting medicine, is not conducive to ventilation and its stimulatory responses to chemical stimuli. The study demonstrates, therefore, that wake-promoting cortical function, as assessed from the EEG and locomotion recordings in the present study, is not congruous with the central regulation of ventilation by the brain. Further, modafinil, showed some suppressive effect on respiratory rate. Yet this effect was rather mild, failing to cause untoward changes in blood gas content. These findings run against our working hypothesis in that, while confirming that modafinil promotes wakefulness, we failed to lend support for the notion of its being augmentative for ventilation and ventilatory reactivity in conscious rodents. This hypothesis was ideated on the premise that cortical function is a key controller of the brain stem respiratory network [26], and integration of hyperventilatory neural inputs: hypercapnic emanating from central medullary chemoreceptors $[27,28]$ and hypoxic emanating from carotid chemoreceptors [29]. The premise was grounded in the fact that wakefulness and locomotion augment ventilation and its reactivity [26] as opposed to natural sleep or anesthetic-induced sleep [30,31]. Thus, the finding of the lack of ventilatory augmentative effect of modafinil at a time of apparent cortical activation was rather unexpected. Our findings suggest the biological plausibility that cortical regulation of wakefulness and respiration proceeds from dichotomy to a possibly merge in lower brain areas where it loses selectivity. Modafinil would then act predominantly on the brain cortex.
The interpretation of the present findings that modafinil, which acts in narcoleptic patients by enhancement of cortical excitability [32, 33], does not promote but might rather suppress ventilation is backed by previous studies on the suprapontine mechanisms in the regulation of respiration in decerebrated and decorticated cats conducted in the 1960-1980s [34, 35]. In those reports, resting ventilation and hypoxic ventilatory response were enhanced in decorticated cats, suggesting that the cortex has a ventilatory suppressive property. Thus, we surmise that modafinil enhance the variability of the breathing pattern in $\mathrm{V}_{\mathrm{T}}$ with promoted wakefulness as mentioned in previous literature [36], but does not facilitate absolute level of ventilation due to its predominantly cortical site of action rather than the brain stem respiratory network that is affected by other traditional wakepromoting drugs $[11,12,14]$. The exact neuronal mechanism underlying the action of modafinil was not the issue of the present study as it requires alternative study designs. The enhanced wakefulness and locomotor activity revealed in the present study after both low and high doses of modafinil has been demonstrated in other studies on the subject $[23,24,37]$. However, the finding of a ventilatory suppressive effect of modafinil, particularly on the hypercapnic response, albeit slight, was fairly unexpected in view of a recent report demonstrating that daily oral modafinil treatment alleviates hypercapnic respiratory failure in COPD patients [10]. The discrepancy might be explicable by different methodological approaches: 1) human vs. rodents, 2) human vs. experimental animal dose (100-200 mg/body vs. 100-200 mg/kg), 3) dosage regimen (daily for 2 weeks vs. one time only), and 4) condition (hypercapnic respiratory failure patients vs. healthy normocapnic animals).

This study has some limitations to be considered. Firstly, one time application of modafinil may not be sufficient for the evaluation of a change in ventilatory control as judged from a report of gradual alleviation of respiratory failure in COPD patients during chronic daily dosing of modafinil [9]. Secondly, the present study was conducted in healthy animals. Deteriorated ventilation in an animal model of respiratory failure could be more affected by modafinil. Modafinil displays cortical enhancing activity in narcoleptic, but not in healthy subjects [27]. Thirdly, we only analyzed short-term ventilatory responses to hypercapnia and hypoxia (5 min recording per each protocol, see Fig. 1). The result of longer term observation might be different, particularly that hypercapnia and hypoxia per se promote wakefulness in short-term; on which background the action of modafinil might be subdued. Fourthly, we used animals of different species, i.e., mice in the measurement of ventilation and rats in blood gas analyses. The findings in both measurements agreed and indicated the absence of 

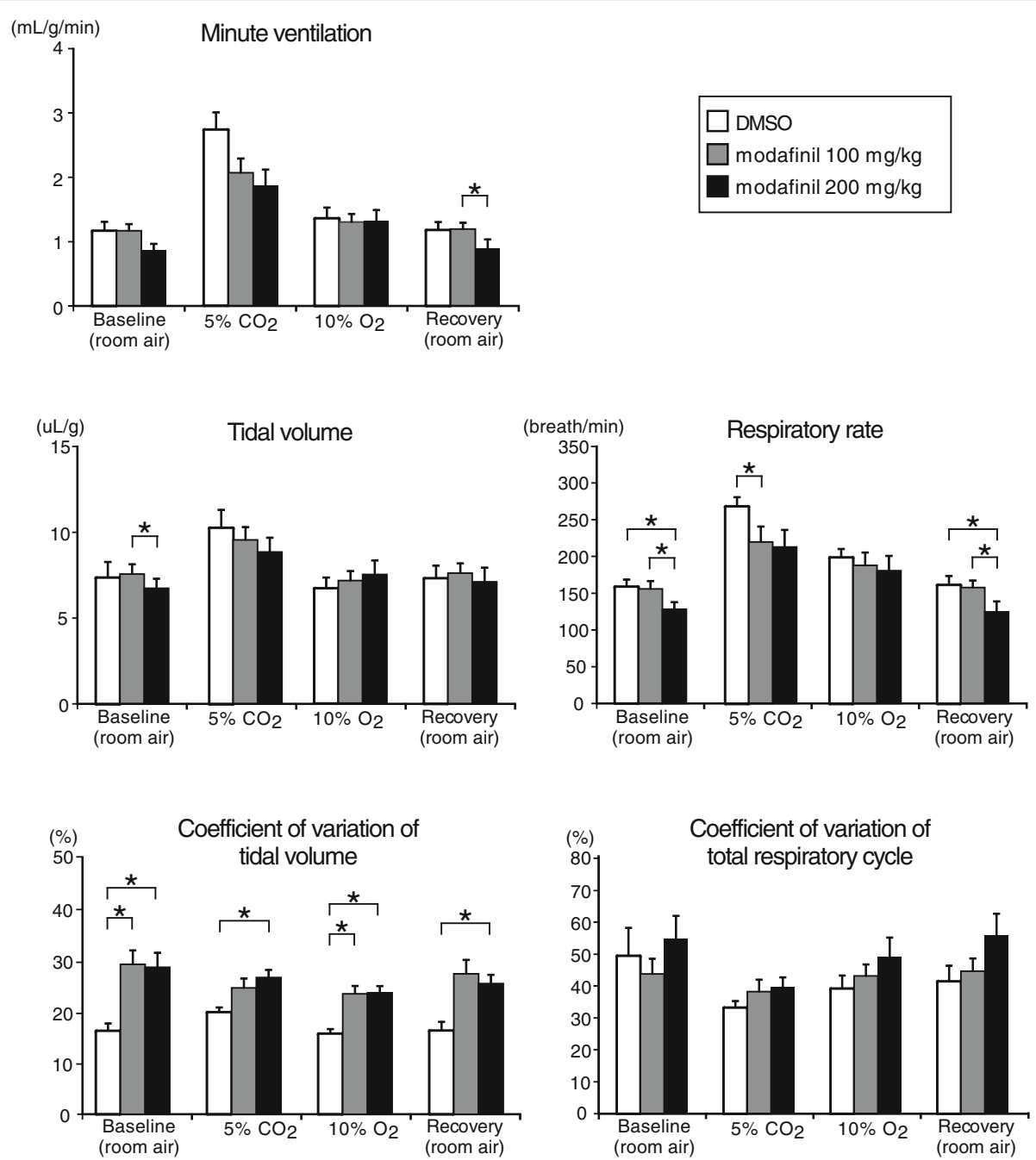

Fig. 4 Minute ventilation $\left(\dot{V}_{E}\right)$, tidal volume $\left(V_{T}\right)$, respiratory rate $(R R)$, and coefficient of variations of $V_{T}$ and total respiratory cycle in the three pharmacological conditions (DMSO and 100 and $200 \mathrm{mg} / \mathrm{kg}$ of the modafinil) in response to the four air conditions (baseline room air, 5\% $\mathrm{CO}_{2}, 10 \% \mathrm{O}_{2}$, and recovery room air). Significant interactions between conditions were revealed by the two-way repeated measures ANOVA for $\dot{V}_{E}(F(6,48)=5.54$, $\left.\varepsilon_{\mathrm{GG}}=0.21, P<0.05\right), \mathrm{V}_{\mathrm{T}}\left(F(6,48)=3.47, \varepsilon_{\mathrm{GG}}=0.39, P<0.05\right)$, and the coefficient of variation of $\mathrm{V}_{\mathrm{T}}(F(6,48)=2.96, P<0.05)$. For $R R$, the interaction was marginally short of conventional level of statistical significance $\left(F(6,48)=2.93, \varepsilon_{G G}=0.38, P=0.07\right)$. Post hoc Bonferroni test revealed some significant differences $(*)$. For the coefficient of variation of total respiratory cycle, either the interaction between conditions or the main effect regarding pharmacological condition was not significant $(F(6,48)=0.85, P=0.85$, and $F(2,16)=2.62, P=0.10$, respectively). DMSO: dimethyl sulfoxide

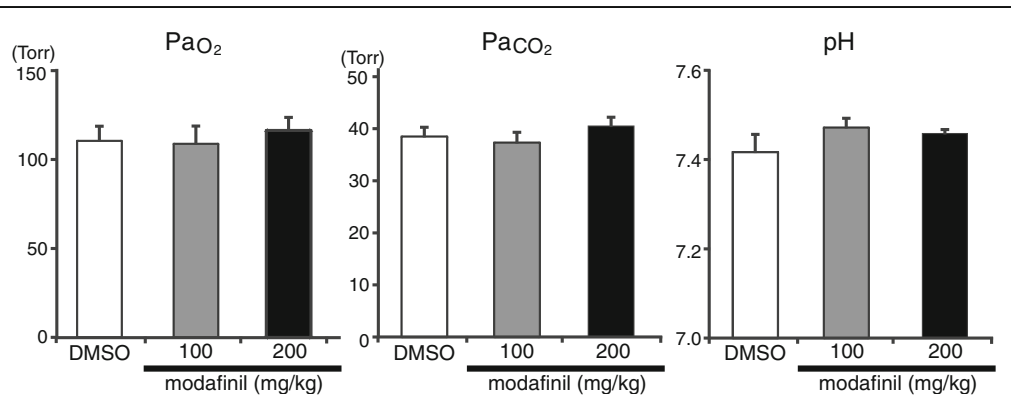

Fig. 5 Blood gas analysis in spontaneous breathing rats at room air in the two pharmacological conditions: DMSO and 100 and 200 mg/kg of the modafinil. No significant differences among the three pharmacological conditions were observed. DMSO: dimethyl sulfoxide 
ventilatory stimulatory action of modafinil. However, higher dose of modafinil slightly suppressed respiratory rate in room air condition in mice but did not affect blood gas in rats. This discrepancy may be explained by species difference and by relative weakness of ventilatory suppressive action of modafinil, as lower dose of modafinil did not affect either respiratory rate, tidal volume, or blood gas. Lastly, concerns may be raised that resting ventilation or the hypoxic ventilatory response may be confounded even without modafinil when hypoxic exposure was repeated. It may occur due to centrally- or muscle fatigue-mediated hypoxic ventilatory depression. However, our pilot study (see Additional file 1) showed that it does not occur at least in our gas exposure protocol. Nonetheless, the conclusion that modafinil does not promote respiration remains robust regardless of the experimental limitations.

The advantage of the present study is that it shows that modafinil is highly selective in its wake-promoting activity and is not concurrently conducive to ventilation and its responsiveness to chemical stimuli of hypercapnia and hypoxia. These findings reinforce clinical safety of modafinil for patients with narcolepsy or other daytime sleepiness, without adverse increase in metabolic rate that could lead to increased ventilation. The disadvantage, however, is that we were unable to unravel another facet of modafinil action, intuitively presumed, i.e., ventilatory facilitation, which could be of clinical interest in view of the paucity of true and safe ventilatory stimulants.

\section{Conclusion}

We conclude that modafinil promotes wakefulness but not ventilation or its responsiveness to chemical stimuli in unanesthetized rodents.

\section{Additional files}

Additional file 1: A pilot study for change in minute ventilation, tidal volume, and respiratory rate after repeated series of hypoxic exposure. Ventilatory parameters (minute ventilation, tidal volume, and respiratory rate) in the resting room air condition and their responses to hypoxia $\left(10 \% \mathrm{O}_{2}\right)$ did not change while hypoxic exposure with vehicle injection (DMSO trial 1-3: DMSO $0.5 \mathrm{\mu L} / \mathrm{g}$ each) was repeated three times. DMSO, dimethyl sulfoxide. (PDF $100 \mathrm{~kb}$ )

Additional file 2: Different behaviors of mice in an ordinary cage observed after administration of modafinil $100 \mathrm{mg} / \mathrm{kg}$ (right cage) vs. DMSO (left cage). The video was recorded 45-50 min after administration of modafinil or DMSO. DMSO: dimethyl sulfoxide. (MP4 $74823 \mathrm{~kb}$ )

\section{Abbreviations}

DMSO: Dimethyl sulfoxide; EEG: Electroencephalogram; RR: Respiratory rate; $\dot{V}_{\mathrm{E}}$ : Minute ventilation; $\mathrm{V}_{\mathrm{T}}$ : Tidal volume

\section{Acknowledgement}

We thank S. Okazaki in Waseda University and T. Matsumura in Chiba University for excellent technical advice concerning the data analyses.

\section{Funding}

This work was supported by JSPS KAKENHI Grant Numbers 24790803, 25540130, 26460311, 26670676 and 15 K00417.

\section{Availability of data and material}

The datasets generated during the current study are available from the corresponding author on reasonable request.

\section{Authors' contributions}

JT designed the study, performed the animal experiments, analyzed data, and drafted the manuscript. IF conducted the surgery of EEG attachment for all mice and performed the all animal experiments. KT performed the statistical analysis, and drafted the manuscript. YH conducted the surgery of arterial catheter insertion for all rats. MP interpreted the results of experiments and revised the manuscript. KT participated in the design of the study. YO participated in the design of the study and revised the manuscript. All authors participated in data interpretation and approved the final manuscript.

\section{Competing interests}

The authors declare that they have no competing interests.

\section{Consent for publication}

Not applicable.

\section{Ethics approval}

All experiments were performed with the approval of the Animal Experiment Ethics Committee of the Murayama Medical Center, and in accordance with the Guiding Principles for the Care and Use of Animals of the Physiological Society of Japan.

\section{Author details}

'Department of Respirology, Graduate School of Medicine, Chiba University, 1-8-1 Inohana, Chuo-ku, Chiba city, Chiba 260-8670, Japan. ${ }^{2}$ Clinical Research Center, Murayama Medical Center, 2-37-1 Gakuen, Musashimurayama, Tokyo 208-0011, Japan. ${ }^{3}$ Department of Biomedical Engineering, Graduate School of Science and Engineering, Toyo University, 2100 Kujirai, Kawagoe, Saitama 350-8585, Japan. ${ }^{4}$ Fujita Memorial Nanakuri Institute, Fujita Health University, 423 Oodori-cho, Tsu, Mie 514-1296, Japan. ${ }^{5}$ Department of Pediatrics, School of Medicine, University of Yamanashi, 1110 Shimokato, Chuo, Yamanashi 409-3898, Japan. ${ }^{6}$ Opole Medical School, 68 Katowicka Street, Opole 45-060, Poland.

Received: 13 July 2016 Accepted: 2 November 2016

Published online: 14 November 2016

\section{References}

1. Randomized trial of modafinil for the treatment of pathological somnolence in narcolepsy. US Modafinil in Narcolepsy Multicenter Study Group. Ann Neurol. 1998;43:88-97.

2. Moldofsky H, Broughton RJ, Hill JD. A randomized trial of the long-term, continued efficacy and safety of modafinil in narcolepsy. Sleep Med. 2000;1:109-16.

3. Randomized trial of modafinil as a treatment for the excessive daytime somnolence of narcolepsy. US Modafinil in Narcolepsy Multicenter Study Group. Neurology. 2000;54:1166-75.

4. Ishizuka T, Murotani T, Yamatodani A. Modanifil activates the histaminergic system through the orexinergic neurons. Neurosci Lett. 2010;483:193-6.

5. Scammell TE, Estabrooke IV, McCarthy MT, Chemelli RM, Yanagisawa M, Miller MS, et al. Hypothalamic arousal regions are activated during modafinil-induced wakefulness. J Neurosci. 2000;20:8620-8.

6. Ishizuka T, Murakami M, Yamatodani A. Involvement of central histaminergic systems in modafinil-induced but not methylphenidate-induced increases in locomotor activity in rats. Eur J Pharmacol. 2008;578:209-15.

7. Ballon JS, Feifel D. A systematic review of modafinil: potential clinical uses and mechanisms of action. J Clin Psychiatry. 2006;67:554-66.

8. Battleday RM, Brem AK. Modafinil for cognitive neuroenhancement in healthy non-sleep-deprived subjects: a systematic review. Eur Neuropsychopharmacol. 2015:25:1865-81.

9. Parnell H, Quirke G, Farmer S, Adeyemo S, Varney V. The successful treatment of hypercapnic respiratory failure with oral modafinil. Int J Chron Obstruct Pulmon Dis. 2014;9:413-9. 
10. Matos P, Esquinas AM. Modafinil: a novel alternative to non-invasive ventilation in hypercapnic respiratory failure? Int J Chron Obstruct Pulmon Dis. 2015;10:711-3.

11. Eldridge FL, Millhorn DE, Waldrop TG, Kiley JP. Mechanism of respiratory effects of methylxanthines. Respir Physiol. 1983;53:239-61.

12. Solt K, Cotten JF, Cimenser A, Wong KF, Chemali JJ, Brown EN. Methylphenidate actively induces emergence from general anesthesia. Anesthesiology. 2011;115:791-803.

13. Mediavilla A, Feria M, Fernandez JF, Cagigas P, Pazos A, Florez J. The stimulatory action of $\mathrm{d}$-amphetamine on the respiratory centre, and its mediation by a central alpha-adrenergic mechanism. Neuropharmacology. 1979;18:133-42.

14. Bairam A, Uppari N, Mubayed S, Joseph V. An Overview on the respiratory stimulant effects of caffeine and progesterone on response to hypoxia and apnea frequency in developing rats. Adv Exp Med Biol. 2015;860:211-20.

15. Javaheri S, Teppema $\sqcup$, Evers JA. Effects of aminophylline on hypoxemia-induced ventilatory depression in the cat. J Appl Physiol (1985). 1988;64:1837-43.

16. Kawai A, Okada Y, Muckenhoff K, Scheid P. Theophylline and hypoxic ventilatory response in the rat isolated brainstem-spinal cord. Respir Physiol. 1995;100:25-32.

17. Fukushi I, Takeda K, Yokota S, Hasebe Y, Sato Y, Pokorski M, et al. Effects of arundic acid, an astrocytic modulator, on the cerebral and respiratory functions in severe hypoxia. Respir Physiol Neurobiol. 2016;226:24-9.

18. Oyamada Y, Murai M, Harada N, Ishizaka A, Okada Y. Age-dependent involvement of ATP-sensitive potassium channel Kir6.2 in hypoxic ventilatory depression of mouse. Respir Physiol Neurobiol. 2008;162:80-4.

19. Pokorski M, Takeda K, Sato Y, Okada Y. The hypoxic ventilatory response and TRPA1 antagonism in conscious mice. Acta Physiol (Oxf). 2014;210:928-38.

20. Takeda K, Pokorski M, Sato Y, Oyamada Y, Okada Y. Respiratory toxicity of dimethyl sulfoxide. Adv Exp Med Biol. 2016;885:89-96.

21. Cameron $Y L$, Merazzi D, Mortola JP. Variability of the breathing pattern in newborn rats: effects of ambient temperature in normoxia or hypoxia. Pediatr Res. 2000;47:813-8.

22. Powell FL, Milsom WK, Mitchell GS. Time domains of the hypoxic ventilatory response. Respir Physiol. 1998;112:123-34.

23. Willie JT, Renthal W, Chemelli RM, Miller MS, Scammell TE, Yanagisawa M, et al. Modafinil more effectively induces wakefulness in orexin-null mice than in wild-type littermates. Neuroscience. 2005;130:983-95.

24. Edgar DM, Seidel WF. Modafinil induces wakefulness without intensifying motor activity or subsequent rebound hypersomnolence in the rat. J Pharmacol Exp Ther. 1997;283:757-69.

25. Mahmoudi J, Farhoudi M, Talebi M, Sabermarouf B, Sadigh-Eteghad S. Antidepressant-like effect of modafinil in mice: evidence for the involvement of the dopaminergic neurotransmission. Pharmacol Rep. 2015;67:478-84.

26. Horn EM, Waldrop TG. Suprapontine control of respiration. Respir Physiol. 1998;114:201-11.

27. Nattie E. $\mathrm{CO}_{2}$, brainstem chemoreceptors and breathing. Prog Neurobiol. 1999:59:299-331

28. Feldman JL, Mitchell GS, Nattie EE. Breathing: rhythmicity, plasticity, chemosensitivity. Annu Rev Neurosci. 2003;26:239-66.

29. Prabhakar NR. Oxygen sensing by the carotid body chemoreceptors. J Appl Physiol (1985). 2000;88:2287-95

30. Joseph V, Pequignot JM, Van Reeth O. Neurochemical perspectives on the control of breathing during sleep. Respir Physiol Neurobiol. 2002;130:253-63.

31. Nunn JF. Effects of anaesthesia on respiration. Br J Anaesth. 1990;65:54-62.

32. Joo EY, Hong SB, Kim HJ, Lim YH, Koo DL, Ji KH, et al. The effect of modafinil on cortical excitability in patients with narcolepsy: a randomized, placebo-controlled, crossover study. Sleep Med. 2010;11:862-9.

33. Nardone R, Bergmann J, Lochner P, Caleri F, Kunz A, Staffen W. Modafinil reverses hypoexcitability of the motor cortex in narcoleptic patients: a TMS study. Sleep Med. 2010;11:870-5.

34. Fink BR, Katz R, Reinhold H, Schoolman A. Suprapontine mechanisms in regulation of respiration. Am J Physiol. 1962;202:217-20.

35. Tenney SM, Ou LC. Ventilatory response of decorticate and decerebrate cats to hypoxia and CO2. Respir Physiol. 1977;29:81-92.

36. Bruce EN. Temporal variations in the pattern of breathing. J Appl Physiol (1985). 1996:80:1079-87.

37. Qu WM, Huang ZL, Xu XH, Matsumoto N, Urade Y. Dopaminergic D1 and D2 receptors are essential for the arousal effect of modafinil. J Neurosci. 2008;28:8462-9.

\section{Submit your next manuscript to BioMed Central and we will help you at every step:}

- We accept pre-submission inquiries

- Our selector tool helps you to find the most relevant journal

- We provide round the clock customer support

- Convenient online submission

- Thorough peer review

- Inclusion in PubMed and all major indexing services

- Maximum visibility for your research

Submit your manuscript at www.biomedcentral.com/submit
) Biomed Central 